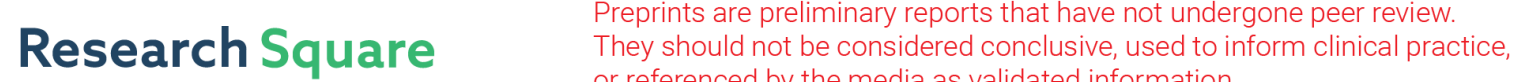 \\ or referenced by the media as validated information. \\ SARS-CoV-2, alcohol consumption, poor social behaviour, and the COVID-19 pandemic
}

Ornelas Marques ( $\nabla$ fomarques@gmail.com )

University of Lisbon

Biological Sciences - Article

Keywords: SARS-CoV-2, COVID-19, alcohol consumption, poor social behavior, pandemic

Posted Date: January 3rd, 2022

DOI: https://doi.org/10.21203/rs.3.rs-1216850/v1

License: (9) This work is licensed under a Creative Commons Attribution 4.0 International License.

Read Full License 


\title{
SARS-CoV-2, alcohol consumption, poor social behavior, and the COVID-19 pandemic
}

\author{
F. O. Marques \\ Retired from the University of Lisbon, Portugal \\ E-mail: fomarques@fc.ul.pt
}

\begin{abstract}
Studies dealing with the SARS-CoV-2 virus and the COVID-19 disease have concentrated on the study of the virus and the disease themselves, but a knowledge of the causes responsible for the pandemic is still lacking. Crucial for effective decision making and control of the pandemic is a thorough and critical analysis of data regarding COVID-19, which shows an intimate relation between the number of cases/deaths and the consumption of alcohol. Ranked top 30 countries in COVID-19 cases/deaths and alcohol consumption coincide, with only a few exceptions. This coincidence is not fortuitous nor surprising, because excessive alcohol consumption is known to have pernicious effects on social behavior, i.e. lead to deviant and irresponsible behavior, which greatly promotes transmission.
\end{abstract}

Keywords: SARS-CoV-2; COVID-19; alcohol consumption; poor social behavior; pandemic

\section{Premises}

COVID-19 is mostly transmitted through the air, thus greatly influenced by proximity between host and recipient. Sneezing, coughing, and kissing without appropriate protection provide the highest 
instantaneous virus load, which is difficult to handle even by a strong immune system.

The number of cases is biased by the number of tests, but to what extent it is not known. Given that the number of asymptomatic people may be large, the number of cases may be greatly underestimated. Therefore, special attention will be paid to the number of deaths attributed to COVID-19, although it may also be somewhat biased.

There are two main kinds of vaccination, natural (infected and recovered, with or without symptoms) and artificial (through injection of classical and MRNA vaccines), but so far the naturally vaccinated people have not been counted as vaccinated.

According to the Dictionary Merriam-Webster, immunity means "the power to keep yourself from being affected by a disease" or "the quality or state of being immune", and immune means "not susceptible". The current vaccines against COVID-19 do not give immunity, they protect from serious consequences of the disease (e.g. death).

Only countries in the northern hemisphere are analyzed here to avoid the mixing of countries with opposite seasonal evolution, i.e. countries currently entering Winter (northern hemisphere with increasing number of cases) or Summer (southern hemisphere with decreasing number of cases). The 7th of December was chosen to avoid the Omicron effect.

Alcohol has negative effects on the social behavior (deviant) and the immune system (deficient, e.g. Molina et al., 2010, and references therein). Misconduct and irresponsible behavior can be responsible for high transmission rates and, therefore, a pandemic.

\section{Analysis of current available data (7 December 2021)}

The analysis of COVID-19 graphs shows that:

(1) COVID-19 is clearly seasonal, with low and high incidences in Summer and Winter, respectively 
(Figs. 1 and S1);

(2) cumulative and daily cases are led by European countries in the top 28 rank (Figs. 1 and S1);

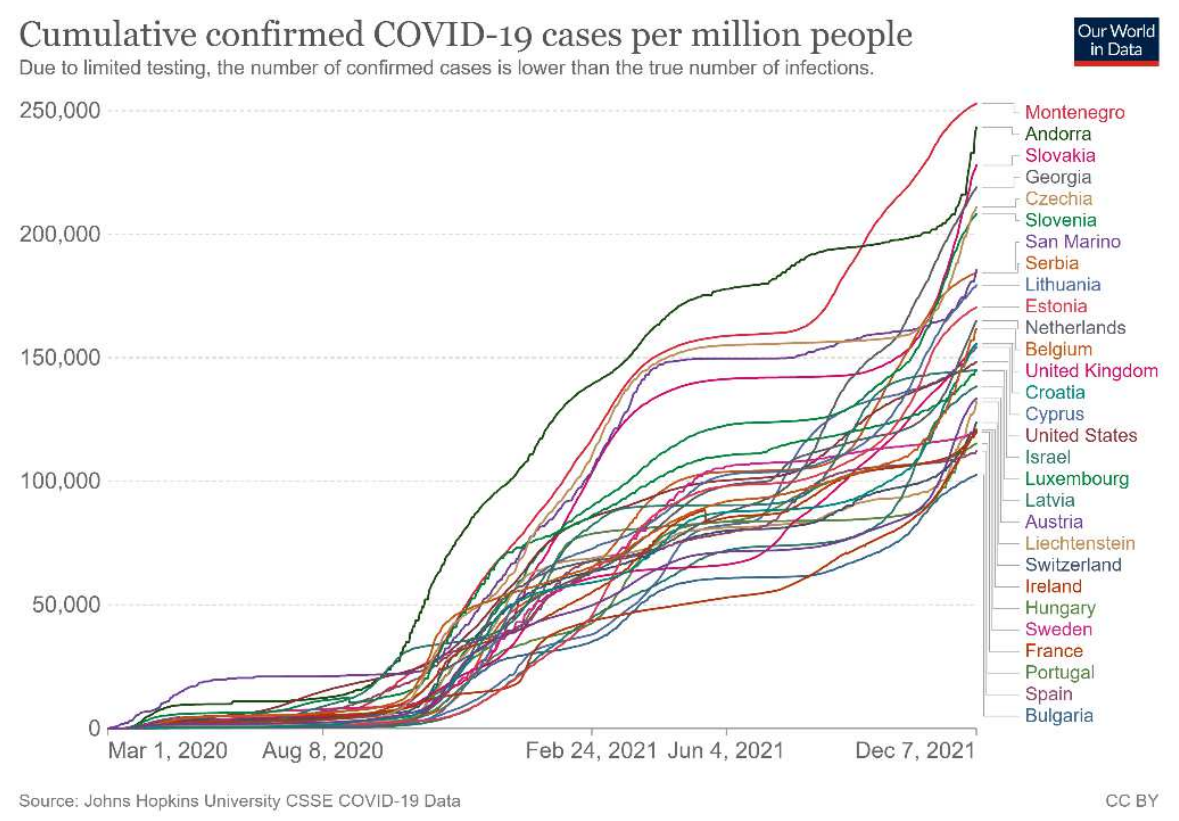

Figure 1. Ranked top 29 countries in the northern hemisphere for cumulative confirmed COVID-19 cases/million people, ranked by total cases/million people. Note that, with a couple of exceptions, all countries are European. Source: Ritchie et al., 2020.

Daily new confirmed COVID-19 cases per million people

7-day rolling average. Due to limited testing, the number of confirmed cases is lower than the true number of infections.

3,500

3,000

2,500

2,000

1,500

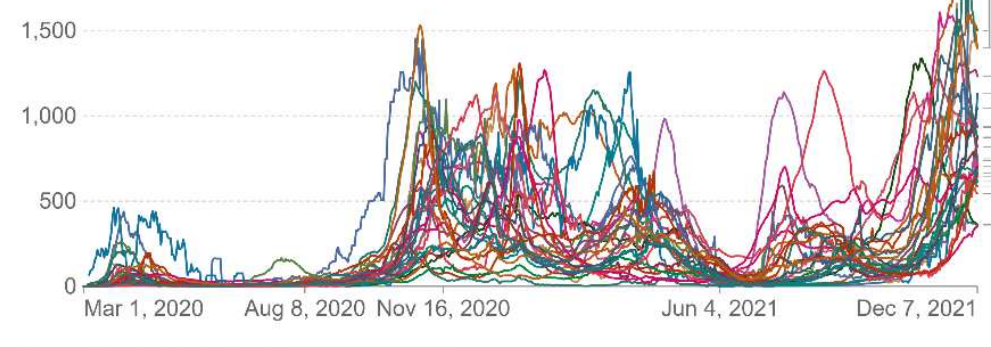

Source: Johns Hopkins University CSSE COVID-19 Data 
Supplementary Figure 1. Ranked top 28 countries in the northern hemisphere for daily new confirmed COVID-19 cases/million people, ranked by daily cases/per million people. Note that all countries are European. Even considering the entire world, the top 23 is composed of European countries. Source:

Ritchie et al., 2020.

(3) regarding cumulative cases by main geographical areas (Fig. S2), the European Union, and North and South Americas have each accumulated almost the triple of cases relative to the World value, and that high-income countries have accumulated about 50 times more cases than low-income countries;

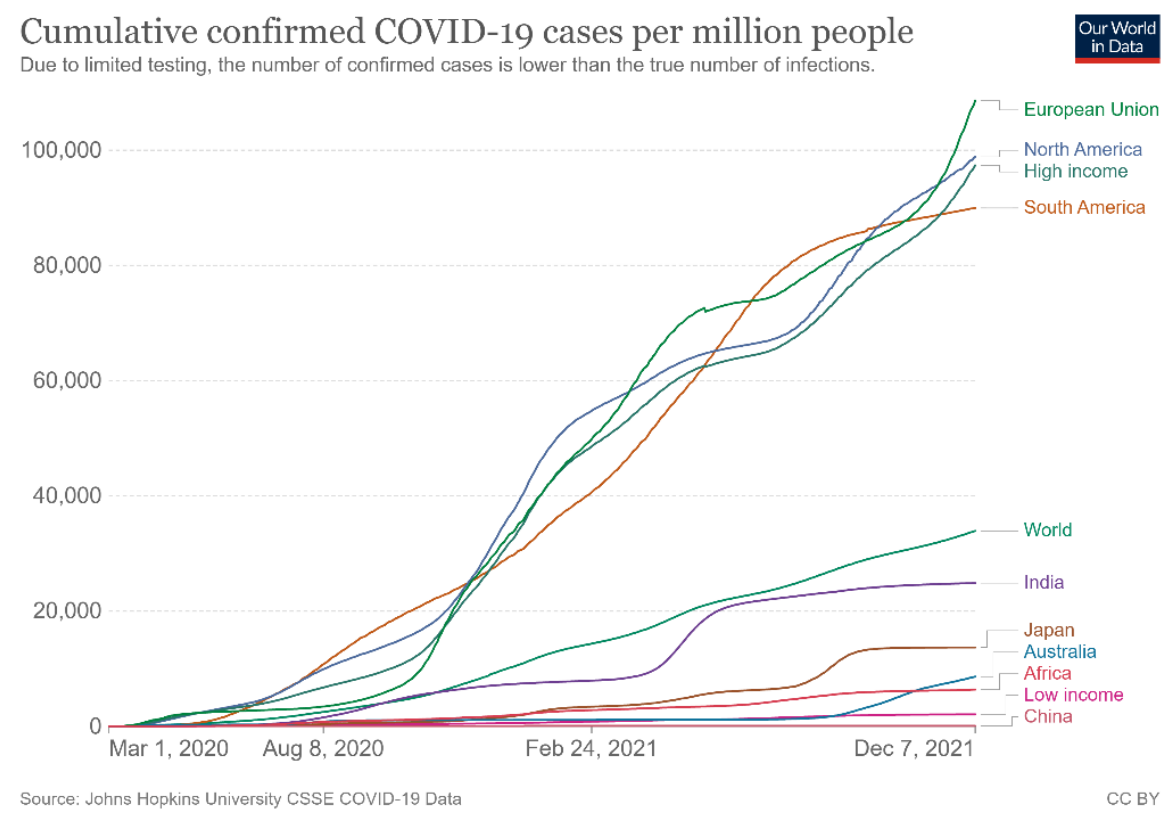

Supplementary Figure 2. Cumulative confirmed COVID-19 cases/million people by main geographical regions. Note that European Union, and North and South Americas have each accumulated almost the triple of cases relative to the World value (large gap on the graph), and that high-income countries have accumulated about 50 times more cases than low-income countries. Source: Ritchie et al., 2020. 


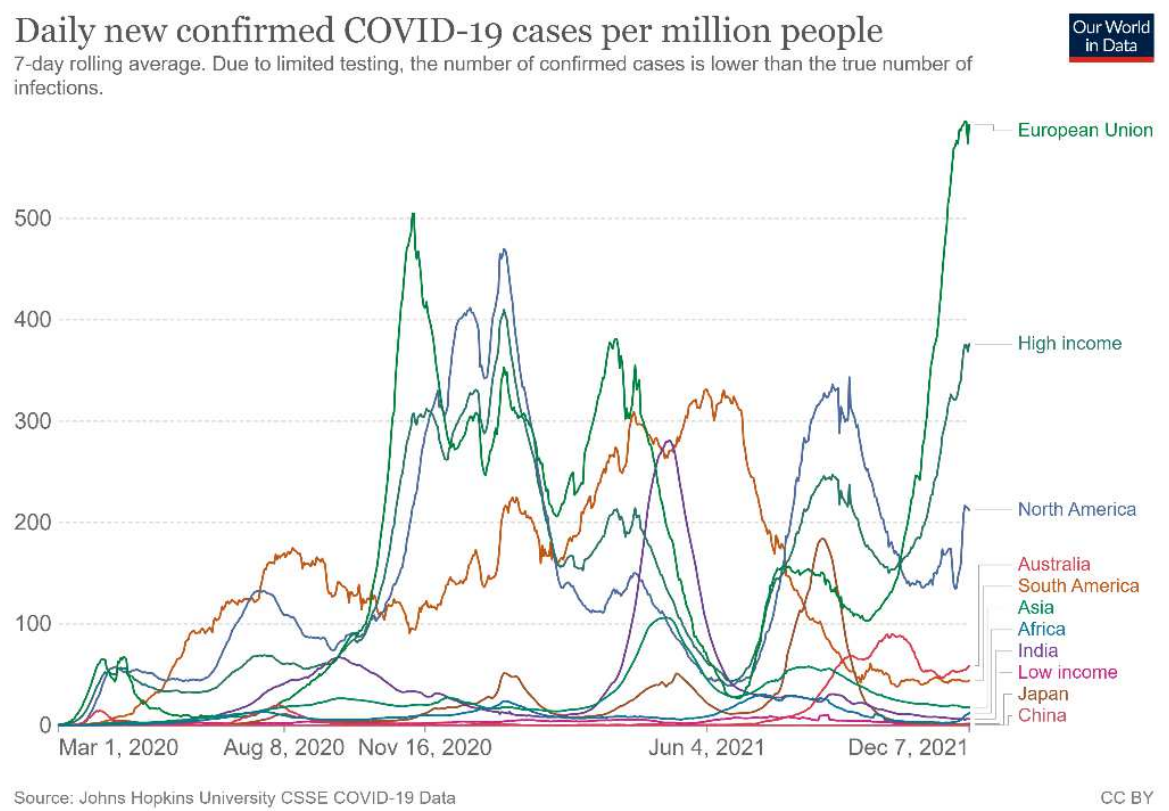

Supplementary Figure 3. Evolution of the daily new confirmed COVID-19 cases/million people by continent, income and selected countries. Note that the European Union currently has almost 3 times more cases than North America, and almost 60 times more than Africa. Source: Ritchie et al., 2020.

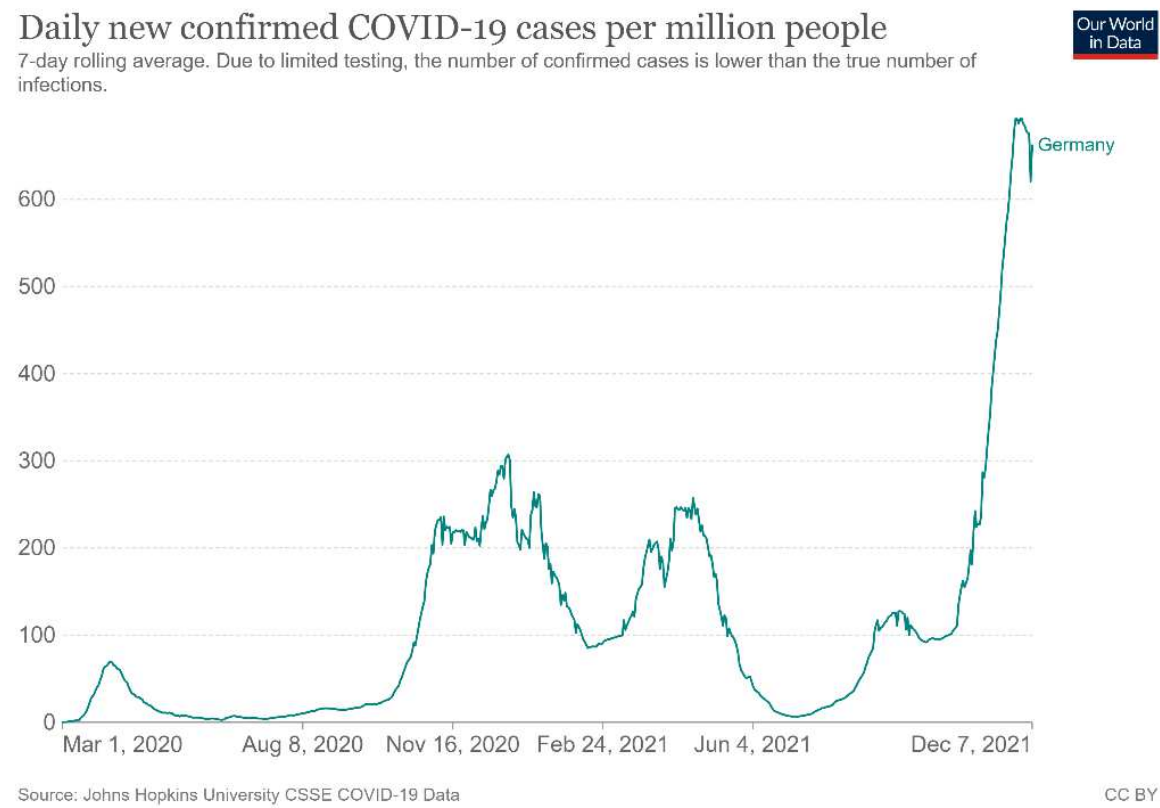

Supplementary Figure 4. Evolution of the daily new confirmed COVID-19 cases/million people in Germany. Note that the number of daily cases has almost tripled relative to the same period in 2020, even though ca. 70\% of the population be fully vaccinated to date. Source: Ritchie et al., 2020. 
(4) as to daily new confirmed COVID-19 cases/million people by continent, income and selected countries (Fig. S3), the European Union has currently almost 3 times more cases than North America, and almost 60 times more than Africa;

(5) regarding the evolution of the daily new confirmed COVID-19 cases/million people in Germany (Fig. S4), the number of daily cases has almost tripled relative to the same period in 2020 , even though ca. $70 \%$ of the population be fully vaccinated to date;

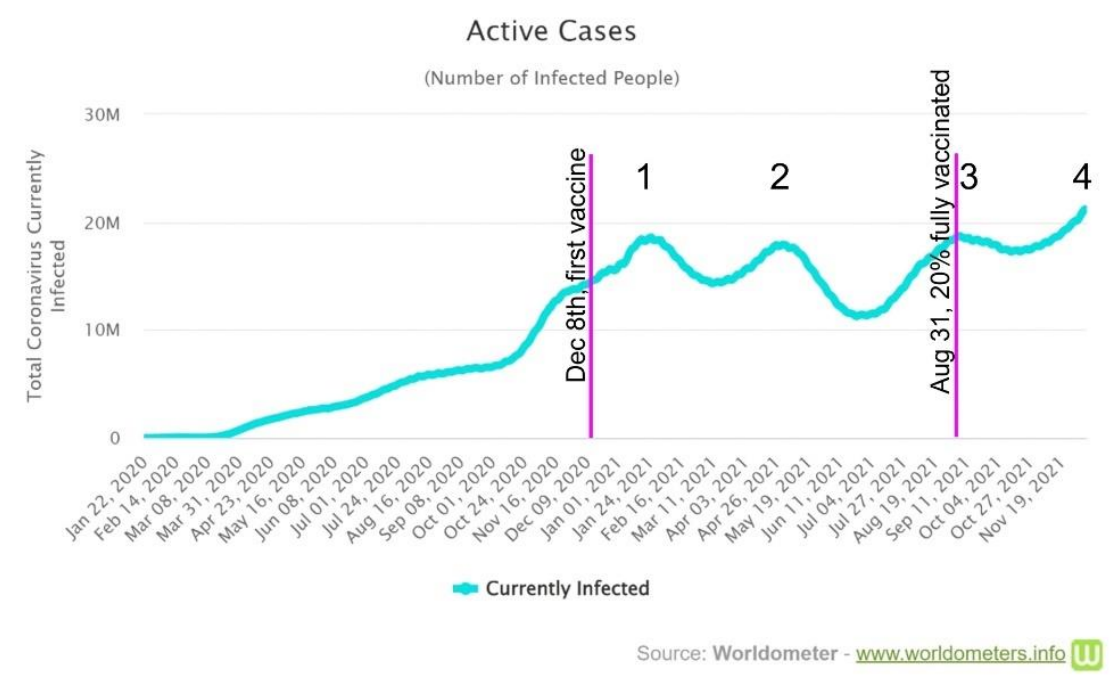

Supplementary Figure 5. Evolution of the number of active cases (cases still waiting for an outcome), which is calculated by removing deaths and recoveries from the total number of cases. Note that: (1) despite some stabilization around 15 M cases, although with fluctuations, after the end of January 2021, the total number of active cases is still increasing and has increased linearly on average; (2) at the end of January 2021, the number of fully vaccinated people was insignificant, therefore vaccination cannot explain the inflection on the curve; (3) from left to right, the three peaks are coincident with winter in the northern hemisphere (peak 1), winter in the southern hemisphere (peak 2), and again winter in the northern hemisphere (recent increase). The evolution of the curve after January 2021 and the recent increase are consistent with the fact that the northern hemisphere dominates de number of cases.

(6) concerning the evolution of the number of active cases (cases still waiting for an outcome) (Fig. S5):

(i) the total number of active cases is still increasing and has increased linearly on average, despite some stabilization with fluctuations around $15 \mathrm{M}$ cases between the ends of January and July 2021; (ii) at the 
end of January 2021, the number of fully vaccinated people was insignificant, therefore vaccination cannot explain the inflection on the curve; (iii) from left to right, the three peaks are coincident with Winter in the northern hemisphere (peak 1), Winter in the southern hemisphere (peak 2), and again Winter in the northern hemisphere (more recent increase, 3 and 4); (iv) the evolution of the curve after January 2021 and the recent increase are consistent with the fact that the northern hemisphere dominates de number of cases.

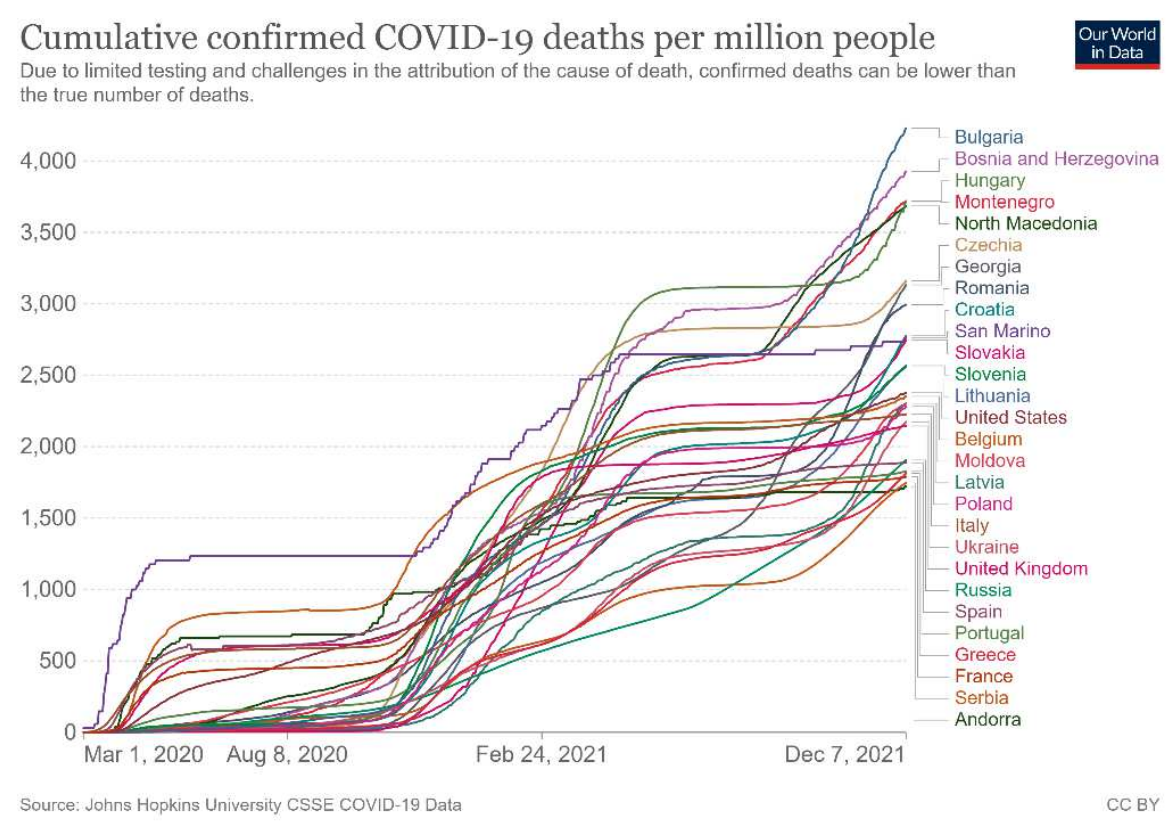

Figure 2. Ranked top 28 countries in the northern hemisphere for cumulative confirmed COVID-19 deaths/million people, ranked by total deaths/million people. Note that the top 28 is composed of European countries, except for the USA, Ukraine and Russia in the bottom half. Top 1 in the world was Peru on the 7th of December 2021. Source: Ritchie et al., 2020.

(7) regarding the cumulative confirmed COVID-19 deaths/million people in the northern hemisphere (Fig. 2), the top 28 is comprised of European countries, with the exception of three countries (USA, Mexico and Russia, all in the bottom half);

(8) as for daily new confirmed COVID-19 deaths/million people in the northern hemisphere (Fig. S6), the top 28 is comprised of European countries, except for the USA; 


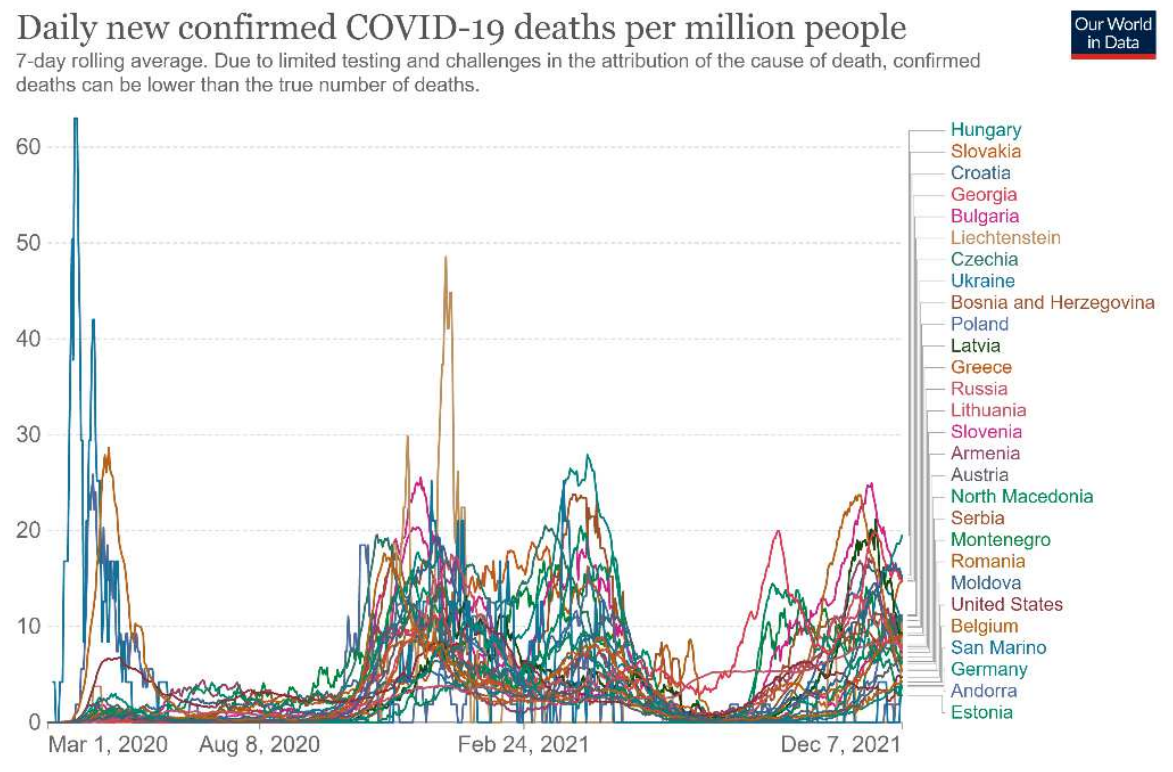

Supplementary Figure 6. Ranked top 28 countries in the northern hemisphere for daily new confirmed COVID-19 deaths/million people, ranked by new deaths/million people. Note that all countries are European except for the USA. Source: Ritchie et al., 2020.

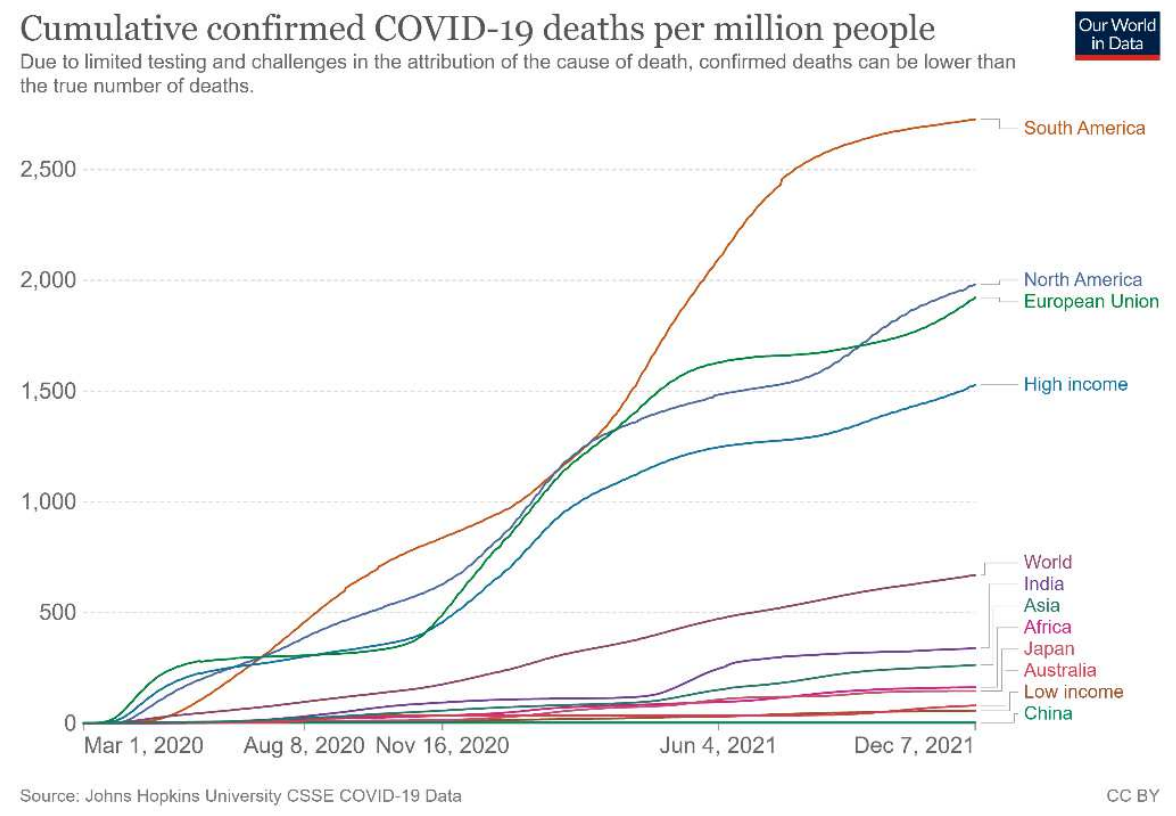

Supplementary Figure 7. Evolution of the cumulative daily new confirmed COVID-19 deaths/million people by continent, income, and selected countries. Note that: (1) South America leads by a significant difference; (2) North America and European Union also show > 4 times difference relative to other main regions of the world; and (3) the difference between high-and low-income countries is about 30 times. Source: Ritchie et al., 2020. 
(9) concerning the daily new confirmed COVID-19 deaths/million people by continent, income, and selected countries (Fig. S7): (i) South America leads by a significant difference, (ii) North America and European Union have more than 4 times difference relative to other main regions of the world, and (iii) the difference between high- and low-income countries is about 30 times;

(10) in all graphs with main geographic regions, Africa, Australia, Japan, China and low-income countries are always at the bottom of the rank.

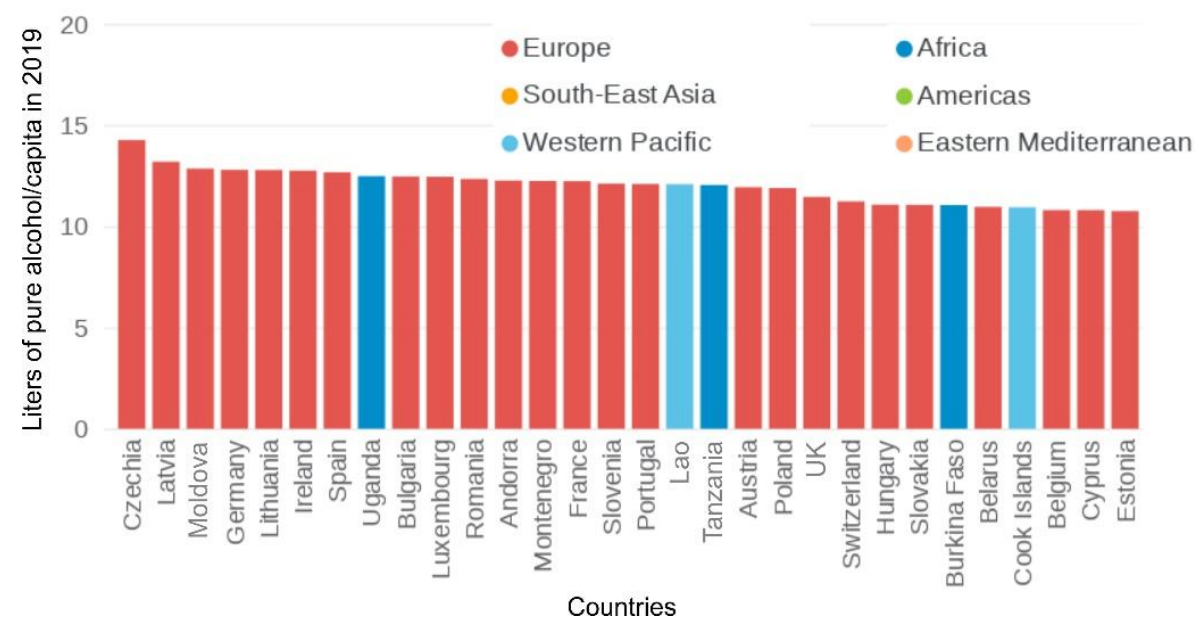

Figure 3. Alcohol consumption in the whole world in 2019, measured in liters of pure alcohol per person $\geq 15$ years of age. The average world consumption was $5.8 \mathrm{l} / \mathrm{person}$. Note that the top 30 is largely dominated by European countries, except for 5 countries from Africa and Western Pacific. Source: World Health Organization (WHO).

\section{Discussion}

Only countries in the northern hemisphere were analyzed here to avoid the mixing of countries with opposite seasonal evolution, which could be thought of as a source of bias. However, the graphs comprising the main regions in the world clearly show that the conclusions reached from the analysis of the northern hemisphere still apply to the whole world. Despite the opposite seasonal evolution between hemispheres, the number of cases/deaths in the northern hemisphere dominate the signal from the seasonal effect in the southern hemisphere. 
At the end of January 2021, the number of fully vaccinated people was insignificant, therefore vaccination cannot explain the inflection on the curve, which could be the result of a combination of seasonal effect (end of Winter) and confinement.

In all graphs comprising main geographic regions, Africa, Australia, Japan, China, and lowincome countries are always at the bottom of the rank, which seems at first sight puzzling because of the mixture of high- and low-income countries. The biased idea that poor countries should be more affected by a pandemic is thus shown to be no more than prejudice. This association should be a matter of urgent and major reflection.

The current available numbers clearly show that Europe faces two major problems: (1) fast contamination as soon as cold arrives, and (2) alcohol consumption, even during Summer, with excessive cases when our immune system is at the peak. The COVID-19 numbers also show that the measures taken by European governments have been mostly ineffective. Media, and, in fact, many decision makers, have mostly been telling people that vaccination is the universal panacea to put an end to the pandemic, and have mostly confused protection with immunity. The (mis)use of the term immunity has serious consequences, mostly because people get the idea that they can do anything (misbehave) because they are "immune". The current vaccines against COVID-19 do not give immunity; they protect from serious consequences of the disease (e.g. death), so avoiding the collapse of the health systems, especially the ICUs (intensive care units). Therefore, the message to be spread to the public is that the vaccine does not give immunity, and that it only protects the vaccinated person, not the people around. In fact, governments that want to make vaccination mandatory in their countries, or even in the whole European Union, are sending the wrong message to the people. A good example is the digital passport, and how people perceive it. It is a passport to misconduct, to be irresponsible, and to legally transmit a virus (again a problem of confusion between the protection given by a vaccine and its power to impede transmission). Current government measures, as in Portugal (European Union), allow people to enter a restaurant with the digital passport, but without a mask and without having to keep the physical distancing. Therefore, the 
main problem, which is irresponsible behavior, will remain unresolved, thus proceeding as a main problem to the next pandemic. The effects of alcohol are twofold: (1) poor and irresponsible social behavior, and (2) destruction of the immune system at different degrees depending on the amount of consumption (e.g. Molina et al., 2010, who concluded that "alcohol abuse suppresses multiple arms of the immune response, leading to an increased risk of infections"). Alcohol and the (wrong) idea that vaccines are a universal panacea have led people to the God/Superman complex, i.e. the feeling that they are immune to anything. First, they are not immune, and second, they are still carriers of the virus and thus able to transmit it.

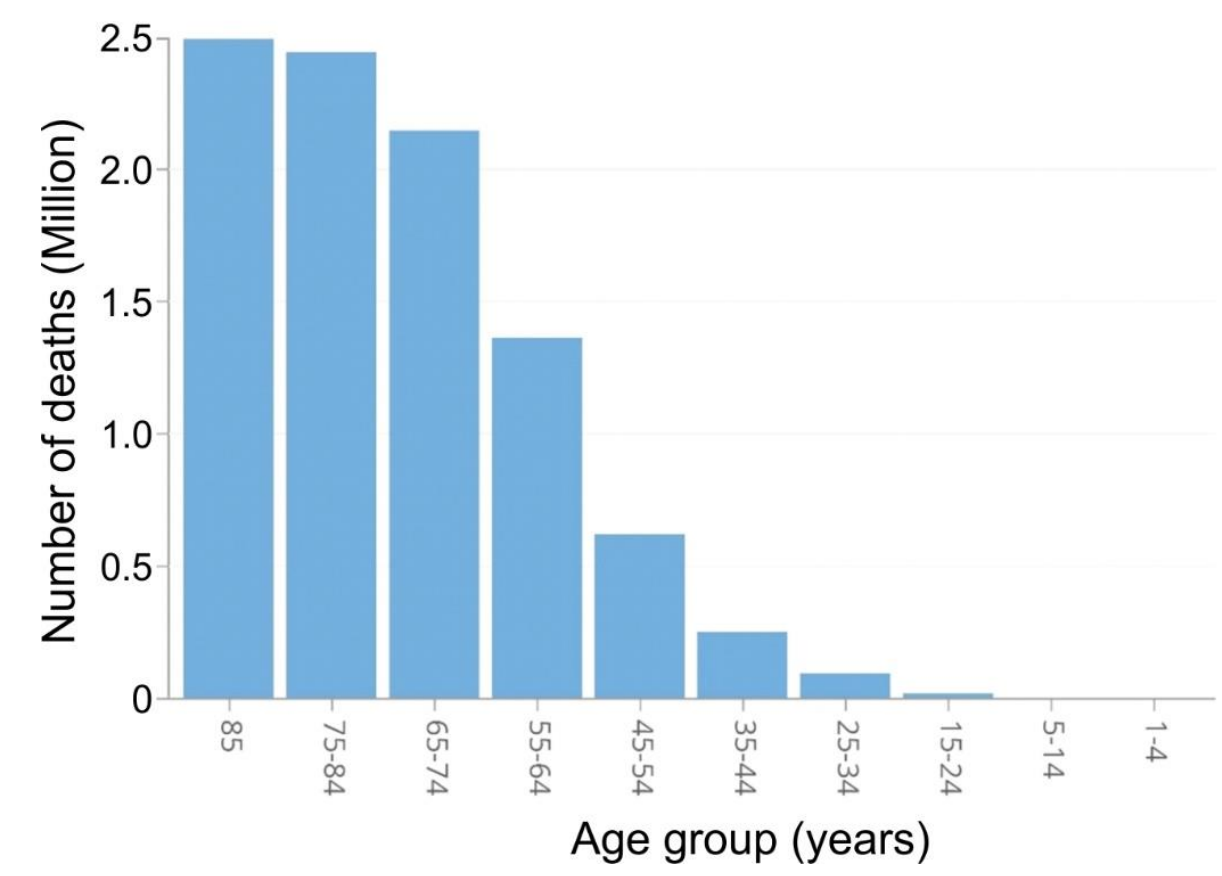

Supplementary Figure 8. Total COVID-19 deaths per age group. Source: data retrieved from https://data.cdc.gov/NCHS/Provisional-COVID-19-Deaths-by-Sex-and-Age/.

The numbers have shown, since the beginning of the COVID-19 pandemic, an exponential relation between death and age (cf. Fig. S8), with very few cases below 10 years and highest number of cases above 64 years. The most obvious conclusion to draw from such a distribution is that young people should avoid, at all costs, the contact with the elderly, unless the youngsters are systematically tested for 
SARS-CoV-2 and, if positive, forbidden to contact elderly people and be immediately quarantined until testing negative.

As shown by the numbers, Europe faces a major problem of alcohol consumption, and this pandemic crisis could be a great opportunity to show people how damaging alcohol consumption can be, especially in terms of behavior in times of a pandemic. Low alcohol consumption triggers the God/Superman complex, which is typically responsible for deviant and irresponsible social behavior. High alcohol consumption is damaging for the health, especially the immune system (Molina et al., 2010). Both represent a major threat at times when we must face a pandemic, because education, a clear mind and a strong immune system are the key to overcome the current and future pandemics.

\section{Messages to take home}

Irresponsible social behavior is the main controlling factor in the virus transmission, which is the main problem when a pandemic is at stake. A deficient immune system is a second stage problem, especially because the number of serious and critical cases may lead to the collapse of National Health Systems and ICUs.

If we do not urgently assume responsible behavior to overcome the current crisis, we will have wasted a great learning opportunity. This time of crisis could be a great opportunity for people to learn how damaging alcohol consumption can be, especially when proper education and conduct are the best solution.

Population protection (social distancing, mask, testing regularly and before mixing young and old people, avoid alcohol and irresponsible behavior) and self-protection (vaccine) are necessary but insufficient to overcome the pandemic.

The universal panacea for the current pandemic is not a vaccine, it is education, knowledge, and 
rational and responsible behavior. Ignorance and irresponsible behavior must be avoided at all cost.

\section{References}

Molina, P.E., Happel, K.I., Zhang, P., Kolls, J.K., Nelson, S., 2010. Focus on: Alcohol and the immune system. Alcohol Res Health 33, 97-108.

Ritchie, H., Mathieu, E., Rodés-Guirao, L., Appel, C., Giattino, C., Ortiz-Ospina, E., Hasell, E., Macdonald,B., Beltekian, D., Roser, M., 2020. Coronavirus Pandemic (COVID-19). Published online at OurWorldInData.org. 